\title{
Ganciclovir/Valganciclovir Prophylaxis Decreases Cytomegalovirus-Related Events and Bronchiolitis Obliterans Syndrome after Lung Transplantation
}

\author{
Corinne Chmiel,' Rudolf Speich, ${ }^{1}$ Markus Hofer, ${ }^{2}$ Detlef Michel, ${ }^{4}$ Thomas Mertens, ${ }^{4}$ Walter Weder, ${ }^{3}$ \\ and Annette Boehler ${ }^{2}$ \\ ${ }^{1}$ Department of Internal Medicine and Clinics of ${ }^{2}$ Pulmonary Medicine and ${ }^{3}$ Thoracic Surgery, University Hospital, Zurich, Switzerland; \\ and ${ }^{4}$ Institute for Virology, University of Ulm, Germany
}

Background. Until recently, cytomegalovirus (CMV) infection represented a major threat to lung transplant recipients. Preliminary studies have shown that antiviral prophylaxis might improve the outcome for these patients.

Methods. We extended our initial pilot trial of prolonged prophylaxis with either oral ganciclovir $(1 \mathrm{~g} 3$ times per day) or valganciclovir (450 mg twice per day). The trial included 96 patients who were at risk for CMV-related events.

Results. CMV prophylaxis resulted in a significant decrease in CMV-related events (i.e., active infection and disease), from $75 \%$ in a control group and for 274 cases from the literature who did not receive prophylaxis to a cumulative incidence of $27 \%(P<.001)$. Only $11 \%$ of the prophylaxis recipients experienced CMV disease $(P<.001)$. Moreover, at 5 years, there was a significant decrease in the rate of bronchiolitis obliterans syndrome, from $60 \%$ to $43 \%(P=.002)$, and an improved rate of survival, from $47 \%$ to $73 \%(P=.036)$, irrespective of the immunosuppressive regimen received. CMV strains with UL97 mutations were recovered from 7 of 12 analyzed cases, but the presence of this mutation had no impact on the severity of CMV disease.

Conclusions. A regimen of prolonged ganciclovir or valganciclovir prophylaxis decreased the rate of active CMV infection and disease, reduced the incidence of bronchiolitis obliterans syndrome, and improved the survival rate. Drug-resistant CMV strains may occur, but such strains appeared to have no impact on the outcome of CMV-related events.

Until some years ago, cytomegalovirus (CMV) represented a major threat to lung transplant recipients. Most early series described a cumulative incidence of CMV-related events (i.e., active infection and disease) of $>50 \%$, and figures of up to $80 \%$ for CMV disease and up to $100 \%$ for active CMV infection have been reported [1-9]. In the first reports, death was not infrequent $[2,3,10]$, and an increased incidence of bronchiolitis obliterans syndrome (BOS) $[5,11,12]$ and serious secondary infections [13] has been suggested.

The first attempts to prevent active CMV infection

Received 10 July 2007; accepted 2 November 2007; electronically published 12 February 2008.

Reprints or correspondence: Dr. Rudolf Speich, Dept. of Internal Medicine, University Hospital, Raemistrasse 100, CH-8091 Zurich, Switzerland (klinspr @usz.unizh.ch)

Clinical Infectious Diseases 2008; 46:831-9

(C) 2008 by the Infectious Diseases Society of America. All rights reserved. $1058-4838 / 2008 / 4606-0008 \$ 15.00$

DOI: $10.1086 / 528689$ and disease after lung transplantation included oral acyclovir $[10,11]$ and short courses of intravenous ganciclovir $[14,15]$, but both regimens proved to be ineffective. After Duncan et al. [11] demonstrated that administration of intravenous ganciclovir for 90 days decreased the incidence of CMV-related events, our group was one of the first to administer oral ganciclovir prophylaxis for an even more prolonged time, and we reported our preliminary findings in 1999 [12]. We demonstrated a significant decrease in the incidence of CMV disease and BOS, lower CMV-related costs, and improved rates of survival. Here, we report our extended experience with a consecutive series of 96 patients.

\section{MATERIALS AND METHODS}

Study population. We prospectively recorded data for 175 consecutive patients who underwent lung transplantation during the period November 1992 through March 2005 at Zurich University Hospital (Zurich, 
Switzerland). Sixty-four CMV-seronegative patients who had received a graft from a CMV-seronegative donor and 7 recipients who died within 30 days after transplantation were excluded from the study, because they were judged not to be at risk for CMV disease. The clinical data for the remaining 104 eligible lung transplant recipients who were at risk of developing CMV-related events are shown in table 1 . The overall mean duration of follow-up ( $\pm \mathrm{SD}$ ) was $4.5 \pm 3.0$ years (range, $0.1-$ 12.4 years).

Ninety-six patients who received CMV prophylaxis were compared with 8 historical control subjects who underwent transplantation before May 1994. To support the relevance of the outcome in our small control population, we performed an exhaustive search of the Medline database and compiled all available articles that contained a full data set for patients who underwent lung transplantation $(n=274)$ during 1982-1999 and who had not received CMV prophylaxis. Data with respect to the incidence of CMV-related events and disease from 4 observational studies [1-4] and from the historical control subjects of 5 nonrandomized trials of ganciclovir prophylaxis [59] were compared with data from the current series. With respect to BOS and survival, data were available for only 139 cases reported in the literature $[4,5,8,9]$.

Study protocol. Our protocol was described in detail in our pilot study [12]. As a consequence of its impressive results, and because a randomized, controlled trial was judged to be un- feasible, we decided to continue use of ganciclovir as a routine prophylaxis regimen.

All 96 consecutive patients who underwent lung transplantation and who were at risk for CMV disease received intravenous ganciclovir ( $5 \mathrm{mg} / \mathrm{kg}$ twice per day) during postoperative days 7-21. Thereafter, patients received oral ganciclovir ( 1 g 3 times per day) until the point at which the prednisone dose had been tapered to $<0.1 \mathrm{mg} / \mathrm{kg}$ per day. Since April 2003, the 25 most recent patients have received valganciclovir (450 mg twice per day orally) instead of ganciclovir. Those patients who were still receiving ganciclovir at that time switched their regimen to valganciclovir $(n=27)$.

Clinical management and immunosuppression. Postoperative management has been described elsewhere [16]. Recipients who were seronegative for CMV received organs from $\mathrm{CMV}$-seronegative donors, if possible; otherwise, they received organs from CMV-seropositive donors, without restrictions, through the entire period. The immunosuppressive regimen originally consisted of prednisone, cyclosporine, and azathioprine; the latter agent was replaced by mycophenolate mofetil in 1999.

In addition to routine clinical, laboratory, functional, and radiological evaluations, all patients were assessed quantitatively for the presence of CMVpp65 antigen in peripheral blood leukocyte specimens at regular intervals (usually twice weekly during the first postoperative month). Thereafter, clinical assess-

Table 1. Characteristics of the literature patients and the current study population.

\begin{tabular}{|c|c|c|c|c|}
\hline \multirow[b]{2}{*}{ Characteristic } & \multirow[b]{2}{*}{$\begin{array}{c}\text { Literature data: }^{a} \\
\text { no receipt of } \\
\text { GCVNCV } \\
(n=274)\end{array}$} & \multicolumn{2}{|c|}{ Current study } & \multirow[b]{2}{*}{$P^{\mathrm{b}}$} \\
\hline & & $\begin{array}{c}\text { No receipt of } \\
\text { GCVNCV } \\
(n=8)\end{array}$ & $\begin{array}{l}\text { Receipt of } \\
\text { GCVNCV } \\
\text { prophylaxis } \\
(n=96)\end{array}$ & \\
\hline Age, mean years & 40 & 42 & 44 & NS \\
\hline Female sex & 49 & 87 & 49 & NS \\
\hline \multicolumn{5}{|l|}{ Diagnosis $^{c}$} \\
\hline Cystic fibrosis, bronchiectasis & 12 & 0 & 33 & \\
\hline Emphysema & 32 & 12 & 29 & \\
\hline Parenchymatous lung disease & 28 & 25 & 18 & \\
\hline Pulmonary hypertension & 19 & 25 & 12 & \\
\hline Lymphangioleiomyomatosis & 2 & 38 & 4 & \\
\hline Redo & 2 & 0 & 4 & \\
\hline Donor age, mean years & NA & 25 & 40 & .003 \\
\hline $\begin{array}{l}\text { CMV-negative recipient of a transplant } \\
\text { from a CMV-positive donor }\end{array}$ & 27 & 50 & 46 & NS \\
\hline \multicolumn{5}{|c|}{$\begin{array}{l}\text { NOTE. Data are percentage of subjects, unless otherwise indicated. CMV, cytomegalovirus; GCVNCV, gan- } \\
\text { ciclovir/valganciclovir; NA, not available; NS, not significant. } \\
\text { a Data compiled from an exhaustive literature search [1-9]. } \\
\text { b Comparison with respect to the present study (e.g., between patients who received and did not receive } \\
\text { prophylaxis). } \\
\text { c In } 5 \% \text { of the literature cases, no specific diagnosis was given. }\end{array}$} \\
\hline
\end{tabular}


Table 2. Outcome of the literature patients and the current study population.

\begin{tabular}{|c|c|c|c|c|}
\hline \multirow[b]{3}{*}{ Outcome } & \multicolumn{3}{|c|}{ Percentage of patients } & \multirow[b]{3}{*}{$P^{\mathrm{b}}$} \\
\hline & \multirow[b]{2}{*}{$\begin{array}{l}\text { Literature data: }^{a} \\
\text { no GCVNCV } \\
(n=274)\end{array}$} & \multicolumn{2}{|c|}{ Current study } & \\
\hline & & $\begin{array}{c}\text { No GCVNCV } \\
(n=8)\end{array}$ & $\begin{array}{c}\text { GCVNCV } \\
\text { prophylaxis } \\
(n=96)\end{array}$ & \\
\hline Active CMV infection at 5 years $^{c}$ & 68 & 75 & 31 & $<.001$ \\
\hline CMV disease at 5 years ${ }^{c}$ & 54 & 75 & 16 & $<.001$ \\
\hline BOS grade 1 at 5 years ${ }^{c, d}$ & 78 & 60 & 43 & .002 \\
\hline Survival at 5 years ${ }^{c, d}$ & 50 & 47 & 73 & .036 \\
\hline $\begin{array}{l}\text { NOTE. BOS, bronchiolitis obliter } \\
\text { valganciclovir. } \\
\text { a Data were compiled from an exhat } \\
\text { b Comparison with respect to the pr } \\
\text { did not receive prophylaxis). } \\
\text { c Cumulative percentages were calc } \\
\text { d For calculation of BOS and surviva } \\
\text { literature }[4,5,8,9] \text {. }\end{array}$ & $\begin{array}{l}\text { Is syndrome; CMV } \\
\text { tive literature search } \\
\text { sent study (e.g., bet } \\
\text { ated by the Kaplan-N } \\
\text { rates, data were ava }\end{array}$ & $\begin{array}{l}\text { cytomegalovirus } \\
\text { [1-9]. } \\
\text { een patients who } \\
\text { eier method. } \\
\text { able for only } 139\end{array}$ & $\begin{array}{l}\text { GCVNCV, ga } \\
\text { eceived and th } \\
\text { atients describ }\end{array}$ & $\begin{array}{l}\text { ciclovir/ } \\
\text { se who } \\
d \text { in the }\end{array}$ \\
\hline
\end{tabular}

ment was performed weekly during the second postoperative month and then every second or third week for the next 4 postoperative months. Surveillance bronchoalveolar lavage and transbronchial lung biopsy specimens were obtained monthly for the first 6 postoperative months, as reported previously [17].

Definitions and study end points. Active CMV infection was defined as identification of the virus by shell-vial or conventional culture of blood, urine, throat swab, or bronchoalveolar lavage fluid specimens (i.e., viral shedding) or by detection of pp65 antigenemia. CMV disease was defined as clinical signs and symptoms plus histologic evidence of pneumonia, colitis, or gastroenteritis, accompanied by a positive viral culture result, detection of CMV by PCR of biopsy specimens, and active CMV infection. CMV-related events were defined as CMV disease and/or active CMV infection. CMV disease was considered to be mild, moderate, or severe on the basis of the duration of hospitalization and whether the patient survived, as follows: mild disease, duration of hospitalization of $<1$ week; moderate disease, duration of hospitalization of 1-3 weeks; and severe disease, duration of hospitalization of $>3$ weeks or death due to CMV-associated disease. Because of progressive increases in pp65 antigen levels and/or presence of symptoms despite continued oral prophylaxis, intravenous ganciclovir treatment was initiated for 9 patients, and subsequent intravenous foscarnet was administered to 1 of these 9 patients. BOS was defined according to the updated criteria of the International Society for Heart and Lung Transplantation [18].

Statistical analysis. Data are expressed as mean $\pm \mathrm{SD}$. Between-group comparisons were performed with the MannWhitney $U$ test for continuous variables and with Fisher's exact test for discrete variables. Survival was calculated by the KaplanMeier cumulative proportion of surviving patients, and be- tween-group differences were determined by the log rank test. Significance was defined as $P<.05$.

\section{RESULTS}

There were 96 consecutive lung transplant recipients at risk of acquiring CMV disease. The patients received ganciclovir or valganciclovir prophylaxis for $507 \pm 398$ days.

The main outcomes for patients and the characteristics of CMV-related events (i.e., active infection and disease) for the present series are shown in tables 2 and 3. Fifteen patients in the prophylaxis group had asymptomatic active CMV infection, with pp65 antigen detected in peripheral blood leukocytes, $299 \pm 245$ days after transplantation. The peak pp65 antigen level was $35 \pm 48$ cells/250,000 leukocytes, and the overall duration was $145 \pm 143$ days, with a prolonged waxing and waning course in 7 patients.

Six (75\%) of 8 lung transplant recipients in the control group developed CMV disease, compared with 11 (11\%) of 96 patients who received prophylaxis $(P<.001)$ (figure $1 A)$. The overall incidence of CMV disease among the patients described in the literature was $56 \%$.

Summing up the cases of active CMV infection and disease resulted in a cumulative incidence of CMV events of 75\% (6 cases) in the historical control group, compared with $27 \%$ (26 cases) in the prophylaxis group $(P<.001)$ (figure $1 B)$. The overall incidence of CMV-related events in our control group was identical to that for the 274 patients described in the literature [1-9], who did not receive any CMV prophylaxis.

Eleven (42\%) of 26 patients who received prophylaxis experienced active CMV infection or disease; these events occurred after the discontinuation of prophylaxis. Treatment con- 
Table 3. Characteristics of cytomegalovirus (CMV) events in the current study population.

\begin{tabular}{|c|c|c|c|}
\hline Characteristic & $\begin{array}{l}\text { Historical control } \\
\quad(n=8)\end{array}$ & $\begin{array}{c}\text { GCVNCV } \\
\text { prophylaxis } \\
(n=96)\end{array}$ & $P$ \\
\hline \multicolumn{4}{|l|}{ Active CMV infection only } \\
\hline All patients & $\ldots$ & 15 & $<.001$ \\
\hline Early antigen appearance, mean postoperative days (range) & NA & $299(69-796)$ & \\
\hline $\begin{array}{l}\text { Early antigen, mean maximum no. of positive cells/250,000 } \\
\text { leukocytes during infection (range) }\end{array}$ & NA & $35(3-189)$ & \\
\hline Duration of early antigen positivity, mean days (range) & NA & $145(15-409)$ & \\
\hline \multicolumn{4}{|l|}{ CMV disease } \\
\hline All patients & 6 & 11 & $<.001$ \\
\hline Diagnosis definite & 6 & 4 & \\
\hline Diagnosis probable & $\ldots$ & 7 & \\
\hline \multicolumn{4}{|l|}{ Main organ involved } \\
\hline Lungs & 5 & 6 & NS \\
\hline Gastrointestinal tract & 1 & 5 & \\
\hline \multicolumn{4}{|l|}{ Severity of disease } \\
\hline Mild & $\ldots$ & 4 & NS \\
\hline Moderate & 1 & 3 & \\
\hline Severe & 5 & 4 & \\
\hline Active CMV infection and disease, overall & 6 & 26 & $<.001$ \\
\hline \multicolumn{4}{|l|}{ Occurrence of active CMV infection or disease } \\
\hline During GCV/NCV prophylaxis & NA & 15 & NA \\
\hline After discontinuation of GCVNCV & NA & 11 & \\
\hline \multicolumn{4}{|l|}{ Management of active CMV infection or disease } \\
\hline Intravenous ganciclovir & 6 & 9 & \\
\hline Continuation of GCVNCV prophylaxis & NA & 9 & .02 \\
\hline Resumption of GCVNCV prophylaxis & NA & 5 & .02 \\
\hline Change from GCV to acyclovir & NA & 1 & .02 \\
\hline None & NA & 2 & \\
\hline $\begin{array}{l}\text { No. of patients with mutations of UL-97 open reading frame of CMV/no. } \\
\text { with wild-type or nondetectable mutations by PCR }\end{array}$ & NA & $7 / 5^{a}$ & \\
\hline Requirement of intravenous GCV, no. of patients with/without mutations & NA & $3 / 2$ & NS \\
\hline
\end{tabular}

NOTE. Data are no. of patients, unless otherwise indicated. GCV, ganciclovir; NA, not available; NS, not significant; VCV, valganciclovir. ${ }^{a}$ CMV genotyping of UL97 was attempted for only the last 12 patients.

sisted of resumption of prophylaxis in 5 of these patients. Four patients required intravenous ganciclovir treatment, and 2 were merely observed. For the 15 patients in whom active CMV infection or disease occurred during prophylaxis, only 5 required intravenous ganciclovir treatment; for the others, prophylaxis was simply continued.

We were able to search for mutations of the UL-97 open reading frame of CMV for the last 12 patients in the current series. In 7 patients, the following mutations in amino acids could be detected: Ala594Ser ( 2 patients), Ala594Leu (1 patient), Ala594Val (1 patient), Lys595Ser (1 patient), and Cys603Try (2 patients). Among patients with CMV disease, the peak pp65 antigen level was higher in patients with a mutation $(136 \pm 127$ cells $/ 250,000$ leukocytes) than in those without a mutation ( $82 \pm 99$ cells/250,000 leukocytes), although the dif- ference was not statistically significant $(P=.22)$. Among the 25 patients who received valganciclovir, only 2 experienced pp65 antigenemia (pp65 antigen level, 5 and 19 positive cells/ 250,000 leukocytes, respectively). There were no mutations detectable in these 2 patients. All other CMV-related events in the prophylaxis group occurred in patients who were receiving ganciclovir $(P=.009)$. Intravenous ganciclovir treatment was used to control CMV infection or disease in only 3 of the 7 patients with known mutations, compared with 2 of the 5 patients who had either wild-type UL97 $(n=2)$ or undetectable UL97 $(n=3)$. The only patient who required intravenous foscarnet treatment, which was administered because of clinical deterioration despite receipt of intravenous ganciclovir, had a documented wild-type UL97 open reading frame.

Patients who received ganciclovir or valganciclovir prophy- 

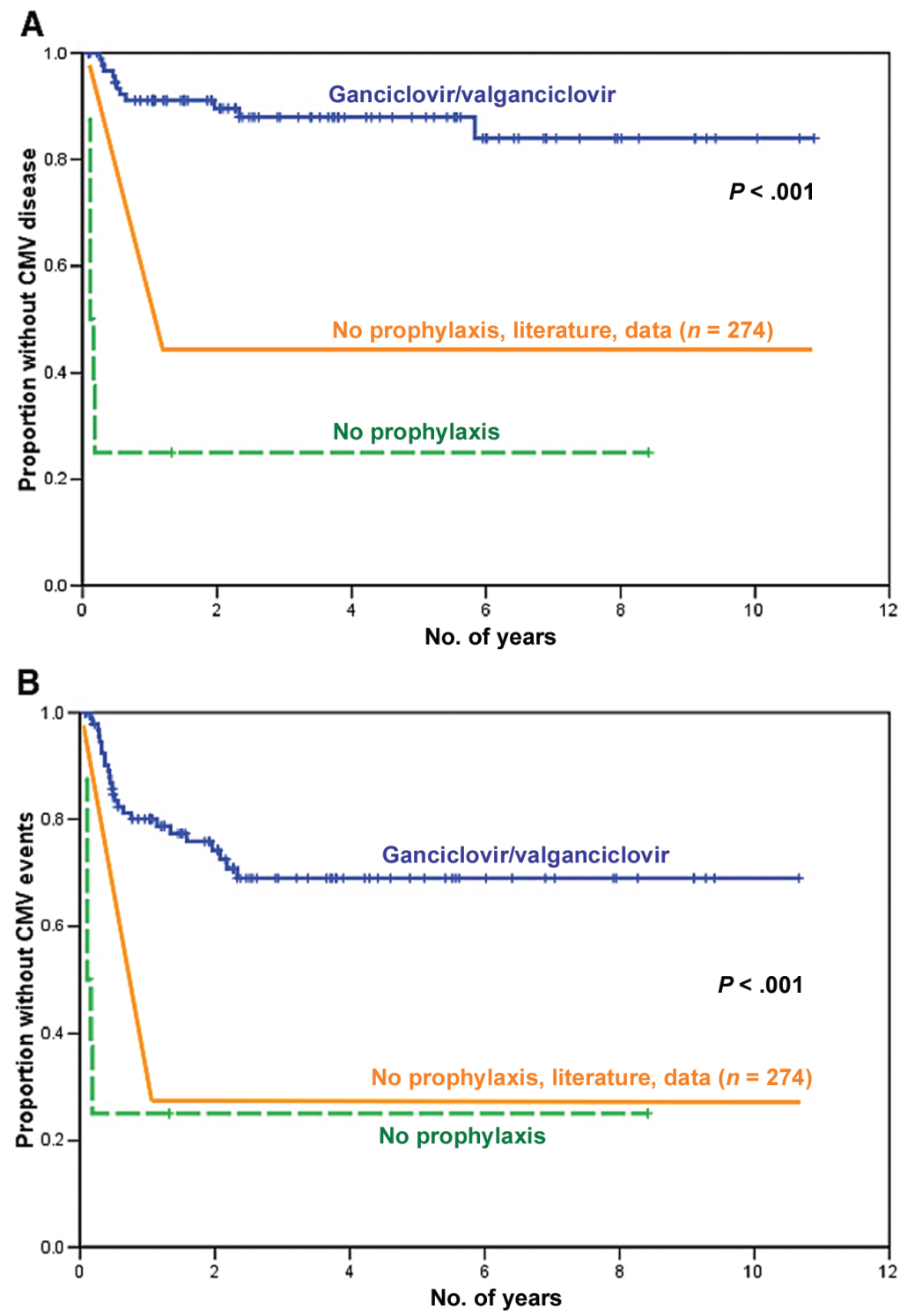

Figure 1. Kaplan-Meier estimates of the likelihood of not experiencing cytomegalovirus (CMV) disease ( $A$ ) or a CMV-related event ( $B$; i.e., CMV infection or disease). $P$ values compare patients receiving prophylaxis with historical control subjects. In addition, data obtained from the literature are shown. CMV prophylaxis with ganciclovir or valganciclovir significantly reduced the cumulative incidence of CMV disease $(A ; P<.001$, by log rank test) and CMV-related events $(B ; P<.001$, by log rank test).

laxis had a significantly lower cumulative incidence of BOS 1 , compared with the historical control subjects (43\% vs. $60 \%$; $P=.002$ ) (figure 2). There was also a significant decrease in the incidence of other BOS stages (data not shown; e.g., for BOS 0 -p, $P=.006$; for BOS $2, P<.001$; and for BOS $3, P=$ $.029)$. The cumulative incidence of patients reaching the composite end point of graft loss due to BOS or death after 5 years was $18 \%$ in the prophylaxis arm versus $50 \%$ in the nonprophylaxis arm (data not shown; $P=.018$ ). With regard to all BOS stages or graft loss due to BOS, there was no difference between ganciclovir recipients and valganciclovir recipients (data not shown).

The rate of survival was significantly improved among patients who received prophylaxis with ganciclovir or valganciclovir, compared with patients who did not received prophylaxis ( $73 \%$ vs. $47 \% ; P=.036$ ) (figure 3 ), and the rate was even better for valganciclovir recipients than for ganciclovir recipients ( $92 \%$ vs. $70 \%$ at 3 years; $P=.029$; data not shown). In a comparison of the outcomes for our historical control group with those for patients described in the literature who had not 


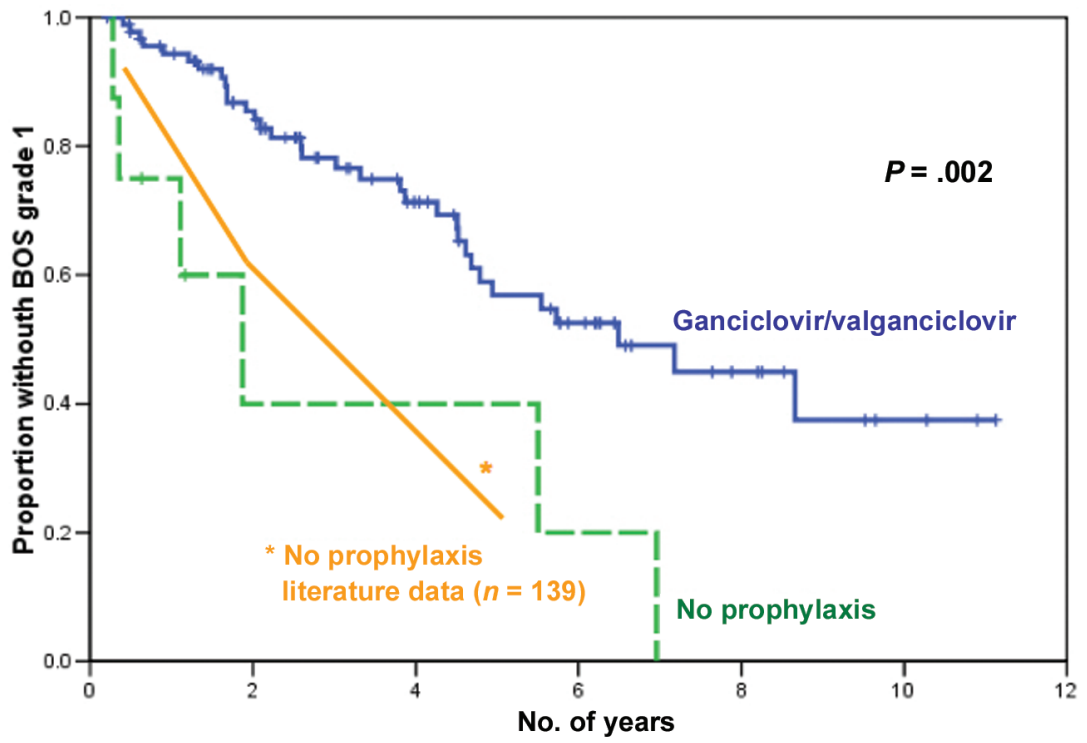

Figure 2. Kaplan-Meier estimates of the likelihood of not experiencing bronchiolitis obliterans syndrome (BOS) grade 1. $P$ values compare patients receiving prophylaxis with historical control subjects. In addition, data from the literature are shown. Cytomegalovirus prophylaxis with ganciclovir or valganciclovir significantly reduced the cumulative incidence of bronchiolitis obliterans syndrome $(P=.002$, by log rank test).

received prophylaxis with ganciclovir, the cumulative incidence of BOS at 3 years was comparable ( $60 \%$ vs. $54 \%$ ), and the rate of survival at 5 years was identical (46\%).

\section{DISCUSSION}

This is, to our knowledge, the largest study to date to show that ganciclovir or valganciclovir prophylaxis significantly decreases the cumulative incidence of CMV-related events, if compared with a historical control group and data from the literature, and to our knowledge, it also has the longest follow-up period. There were no CMV-related deaths, and only 4 of 96 patients in the study group experienced severe CMV disease, compared with 5 episodes of severe disease among 8 historical control patients. In addition, our regimen of CMV prophylaxis resulted in a significant reduction in the cumulative incidence of all BOS (of all stages) and of graft loss due to BOS, as well as a significant improvement in overall survival.

In consideration of the fact that, without prophylaxis, CMV causes serious disease in approximately one-half of lung transplant recipients (occasionally involving a fatal outcome), all lung transplantation centers now have adopted use of some kind of prophylactic regimen. Since the advent of oral ganciclovir and its prodrug valganciclovir, most centers have chosen these drugs as their prophylactic mainstay.

There is, however, uncertainty about the optimal duration of prophylaxis. In our opinion, there is currently a compelling body of evidence that the minimum duration of administration of ganciclovir or valganciclovir in at-risk lung transplant recipients is at least 180 days after transplantation or until the prednisone dosage is tapered to $0.1 \mathrm{mg} / \mathrm{kg}$ per day, as in the present study. The first attempts at preventing CMV disease among lung transplant recipients consisted of administration of intravenous ganciclovir for 3 [14], 6 [15], or 12 [6, 19] weeks, but all regimens failed to show any benefit. The same was true in the study by Weill et al. [20], who administered intravenous ganciclovir, followed by oral ganciclovir, for 6-12 weeks, with the addition of intravenous immune globulin for more than one-half of the patients. Also, Soghikian et al. [5] demonstrated improvement in survival associated with a reduction in the severity of CMV disease with receipt of a regimen of intravenous ganciclovir until day 35 or until the prednisone dose had reached a baseline of $0.2 \mathrm{mg} / \mathrm{kg}$ per day (compared with $0.1 \mathrm{mg} / \mathrm{kg}$ per day in the present study), although they experienced an unacceptably high cumulative incidence of CMV diseases (54\%). This is in line with the findings of Humar et al. [21], who reported an incidence of active CMV infection and disease of $43 \%$ and $19 \%$, respectively, with administration of a 12-week prophylactic course of either ganciclovir (1 g 3 times per day) or valganciclovir (900 mg once per day); the difference between the 2 regimens was not significantly different. On the other side of the spectrum, Palmer et al. [8] reported a reduced incidence of active CMV infection and disease-from $65 \%$ and $29 \%$, respectively, to $35 \%$ and $5 \%$, respectively-associated with prophylaxis involving oral ganciclovir (1 g 3 times per day) continued indefinitely. Recently, Zamora et al. [22] nicely addressed the issue of duration of CMV prophylaxis in a comparison of 5 regimens that were administered anywhere from $<100$ days to up to 365 days; they 


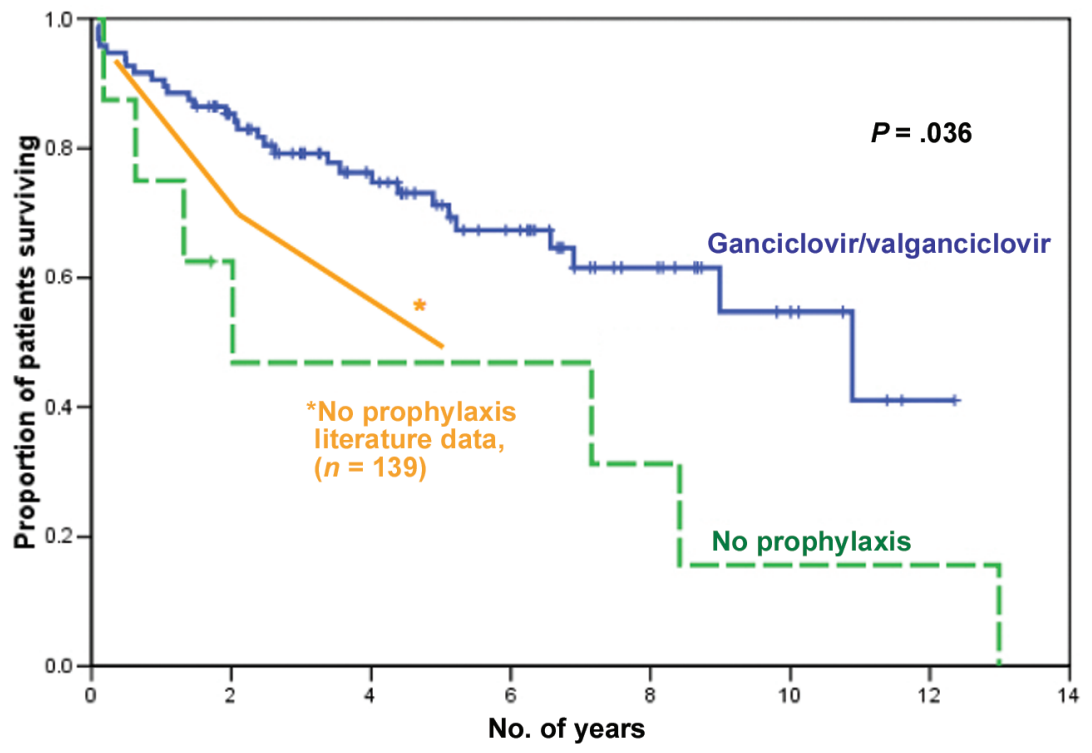

Figure 3. Kaplan-Meier estimates of the likelihood of survival. $P$ values compare patients receiving prophylaxis with historical control subjects. In addition, data from the literature are shown. Cytomegalovirus prophylaxis with ganciclovir or valganciclovir significantly improved the cumulative survival rate $(P=.036$, by log rank test).

found that prophylaxis should be continued for at least 180 days after transplantation to significantly reduce the incidence of CMV disease. However, the number of patients receiving the various prophylactic regimens was rather small. All of these data stand in contradistinction with the study by the colleagues from Papworth [9], who achieved a spectacularly low incidence of $4 \%$ for cases of CMV disease after adopting a regimen of 12 weeks of oral ganciclovir. The reason for this discrepancy is not quite clear, but it has to be mentioned that, in that study, the percentage of CMV-seronegative recipients of grafts from CMV-seropositive donors was only $17 \%$ of all patients who received prophylaxis, compared with $46 \%$ in the present study. In addition, those authors described a high cumulative incidence of BOS at 5 years of $\sim 80 \%$, and CMV prophylaxis did not influence this figure significantly. Thus, it may be argued that this research group tapered immunosuppressive therapy more rapidly than did other research groups, resulting in a low occurrence of CMV disease but, conversely, a high incidence of BOS.

The mean duration of CMV prophylaxis was 507 days (median, 364 days; range, 113-2024 days), which was quite high. The reason for this prolongation of our prophylactic regimen was because (1) almost one-half of CMV-related events occurred after discontinuation of prophylaxis (making the team members reluctant to stop ganciclovir/valganciclovir prophylaxis too early), (2) our very slow tapering regimen of steroids, as well as (3) the continuation of prophylaxis in cases with recurrent acute rejection episodes or BOS, necessitating increased immunosuppression. Although we demonstrated, in our pilot trial, that prophylaxis with a median duration of 210 days (and of up to 320 days) is a cost-effective approach, the cost-effectiveness of the significantly longer period of prophylaxis in this extended series remains to be confirmed.

An important finding of the current study is the fact that CMV prophylaxis both significantly reduced the occurrence of BOS and improved overall survival, and this was independent of the changes in the immunosuppressive regimens over recent years. The decrease in the incidence of BOS is in line with earlier [5, 11, 12] and recent findings [23]. Thus far, to our knowledge, only our pilot trial [12] and 3 other studies [5, 8, 23] have demonstrated an improved rate of survival. Most of the remaining studies either did not mention these 2 outcome parameters $[7,20,21]$, had a follow-up period that was too short [22], or found no difference in the occurrence of BOS and survival [9]. (A criticism of the latter study was already discussed above.) In the current study, although there was no difference between regimens with regard to the occurrence of BOS, the rate of survival was even better among valganciclovir recipients than among ganciclovir recipients. This finding cannot be explained by the current data.

A caveat for prolonged prophylaxis with ganciclovir or valganciclovir may be the fear that resistant CMV strains may arise. However, in a recent study, all CMV strains obtained from 49 patients with antigenemia underwent UL97 sequencing, and only 3 drug-resistant strains were found [21]. In 2 of these cases, foscarnet was necessary to control CMV infection. In the current study, a CMV mutation could be found in 7 of 12 patients in whom CMV UL97 gene sequencing was attempted. The occurrence of a mutation, however, had no clinical impact in our series; for example, the requirement of in- 
travenous ganciclovir was the same for patients with and the patient without a mutation. The single patient who had to be treated with foscarnet had a documented UL97 wild-type CMV strain.

Interestingly, only 2 of 25 valganciclovir recipients had lowlevel, asymptomatic pp65 antigenemia. All other CMV-related events_-including all events in persons with UL97 mutationsoccurred among recipients of ganciclovir prophylaxis $(P=$ .009). Because the overall level of drug exposure is only $60 \%$ for daily 3-g doses of ganciclovir, compared with 900-mg doses of valganciclovir [24], it is possible that the lower drug levels resulting from receipt of oral ganciclovir predispose patients to selection of low-grade UL97 resistance mutations and to later accumulation of other mutations and, thus, greater resistance [25].

A drawback of the present study is its design with use of a historical control group. However, because of the impressive results of our pilot trial, a randomized study was considered not to be ethical. In addition, the data of our small control group with respect to $\mathrm{CMV}$ events, BOS, and survival were enhanced by the comparison with 274 literature cases that did not receive CMV prophylaxis. Moreover, it has to be emphasized that, thus far, there have been no randomized studies comparing antiviral agents and placebo after lung transplantation, and such a trial probably will never be performed.

Preemptive treatment may be an attractive approach. However, a recent study revealed that 5 of 19 patients developed CMV disease, which was not predicted by antigenemia [26]. This unsatisfactory sensitivity may also occur if PCR is usedespecially for gastrointestinal CMV disease, for which the sensitivity of PCR may be as low as 50\% [27]. In addition, it has to be underscored that our control group in fact had been treated with a preemptive approach involving weekly (and, later, biweekly) determinations of the CMVpp65 antigen level.

In conclusion, we can confirm the results from other studies that have found a decrease in active CMV infection and disease using a regimen of prolonged prophylaxis with ganciclovir or valganciclovir. Moreover, there was a decrease in the occurrence of BOS and an improved survival rate that was not attributable to newer immunosuppressive therapies. Drug-resistant CMV strains may arise, but they do not seem to be of clinical relevance in these patients. Because the duration of our prophylactic regimen was longer than that of previous studies, its costeffectiveness needs to be addressed in the future.

\section{Acknowledgments}

Potential conflicts of interest. R.S. has received a free unrestricted educational grant from Roche Pharma Schweiz. All other authors: no conflicts.

\section{References}

1. Duncan AJ, Dummer JS, Paradis IL, et al. Cytomegalovirus infection and survival in lung transplant recipients. J Heart Lung Transplant 1991; 10:638-46.

2. Maurer JR, Tullis DE, Scavuzzo M, Patterson GA. Cytomegalovirus infection in isolated lung transplantations. J Heart Lung Transplant 1991; 10:647-9.

3. Smyth RL, Scott JP, Borysiewicz LK, et al. Cytomegalovirus infection in heart-lung transplant recipients: risk factors, clinical associations, and response to treatment. J Infect Dis 1991; 164:1045-50.

4. Ettinger NA, Bailey TC, Trulock EP, et al. Cytomegalovirus infection and pneumonitis: impact after isolated lung transplantation. Washington University Lung Transplant Group. Am Rev Respir Dis 1993; 147:1017-23.

5. Soghikian MV, Valentine VG, Berry GJ, Patel HR, Robbins RC, Theodore J. Impact of ganciclovir prophylaxis on heart-lung and lung transplant recipients. J Heart Lung Transplant 1996; 15:881-7.

6. Halme M, Lautenschlager I, Halme L, Tukiainen P, Mattila S. Ganciclovir prophylaxis after lung and heart-lung transplantation. Transpl Int 1998; 11(Suppl 1):499-501.

7. Brumble LM, Milstone AP, Loyd JE, et al. Prevention of cytomegalovirus infection and disease after lung transplantation: results using a unique regimen employing delayed ganciclovir. Chest 2002; 121: 407-14.

8. Palmer SM, Grinnan DC, Diane Reams B, et al. Delay of CMV infection in high-risk CMV mismatch lung transplant recipients due to prophylaxis with oral ganciclovir. Clin Transplant 2004; 18:179-85.

9. Perreas KG, McNeil K, Charman S, Sharples LD, Wreghitt T, Wallwork J. Extended ganciclovir prophylaxis in lung transplantation. J Heart Lung Transplant 2005; 24:583-7.

10. Duncan SR, Paradis IL, Dauber JH, Yousem SA, Hardesty RL, Griffith BP. Ganciclovir prophylaxis for cytomegalovirus infections in pulmonary allograft recipients. Am Rev Respir Dis 1992; 146:1213-5.

11. Duncan SR, Grgurich WF, Iacono AT, et al. A comparison of ganciclovir and acyclovir to prevent cytomegalovirus after lung transplantation. Am J Respir Crit Care Med 1994; 150:146-52.

12. Speich R, Thurnheer R, Gaspert A, Weder W, Boehler A. Efficacy and cost effectiveness of oral ganciclovir in the prevention of cytomegalovirus disease after lung transplantation. Transplantation 1999; 67: 315-20.

13. Husni RN, Gordon SM, Longworth DL, et al. Cytomegalovirus infection is a risk factor for invasive aspergillosis in lung transplant recipients. Clin Infect Dis 1998; 26:753-5.

14. Bailey TC, Trulock EP, Ettinger NA, Storch GA, Cooper JD, Powderly WG. Failure of prophylactic ganciclovir to prevent cytomegalovirus disease in recipients of lung transplants. J Infect Dis 1992; 165:548-52.

15. Kelly JL, Albert RK, Wood DE, Raghu G. Efficacy of a 6-week prophylactic ganciclovir regimen and the role of serial cytomegalovirus antibody testing in lung transplant recipients. Transplantation 1995; 59:1144-7.

16. Speich R, Boehler A, Zalunardo M, Stocker R, Russi EW, Weder W. Improved results after lung transplantation-analysis of factors. Swiss Med Wkly 2001; 131:238-45.

17. Boehler A, Vogt P, Zollinger A, Weder W, Speich R. Prospective study of the value of transbronchial lung biopsy after lung transplantation. Eur Respir J 1996; 9:658-62.

18. Estenne M, Maurer JR, Boehler A, et al. Bronchiolitis obliterans syndrome 2001: an update of the diagnostic criteria. J Heart Lung Transplant 2002;21:297-310.

19. Hertz MI, Jordan C, Savik SK, et al. Randomized trial of daily versus three-times-weekly prophylactic ganciclovir after lung and heart-lung transplantation. J Heart Lung Transplant 1998; 17:913-20.

20. Weill D, Lock BJ, Wewers DL, et al. Combination prophylaxis with ganciclovir and cytomegalovirus (CMV) immune globulin after lung 
transplantation: effective CMV prevention following daclizumab induction. Am J Transplant 2003; 3:492-6.

21. Humar A, Kumar D, Preiksaitis J, et al. A trial of valganciclovir prophylaxis for cytomegalovirus prevention in lung transplant recipients. Am J Transplant 2005; 5:1462-8.

22. Zamora MR, Nicolls MR, Hodges TN, et al. Following universal prophylaxis with intravenous ganciclovir and cytomegalovirus immune globulin, valganciclovir is safe and effective for prevention of CMV infection following lung transplantation. Am J Transplant 2004; 4: $1635-42$.

23. Ruttmann E, Geltner C, Bucher B, et al. Combined CMV prophylaxis improves outcome and reduces the risk for bronchiolitis obliterans syndrome (BOS) after lung transplantation. Transplantation 2006; 81 : 1415-20.

24. Wiltshire H, Hirankarn S, Farrell C, et al. Pharmacokinetic profile of ganciclovir after its oral administration and from its prodrug, valganciclovir, in solid organ transplant recipients. Clin Pharmacokinet 2005; 44:495-507.

25. Chou S, Waldemer RH, Senters AE, et al. Cytomegalovirus UL97 phosphotransferase mutations that affect susceptibility to ganciclovir. J Infect Dis 2002; 185:162-9.

26. Kelly J, Hurley D, Raghu G. Comparison of the efficacy and cost effectiveness of pre-emptive therapy as directed by CMV antigenemia and prophylaxis with ganciclovir in lung transplant recipients. J Heart Lung Transplant 2000; 19:355-9.

27. Mori T, Mori S, Kanda Y, et al. Clinical significance of cytomegalovirus (CMV) antigenemia in the prediction and diagnosis of CMV gastrointestinal disease after allogeneic hematopoietic stem cell transplantation. Bone Marrow Transplant 2004; 33:431-4. 